\title{
Dual-task interference in visual working memory: A limitation in storage capacity but not in encoding or retrieval
}

\author{
Daryl Fougnie And René Marois \\ Vanderbilt University, Nashville, Tennessee
}

\begin{abstract}
The concurrent maintenance of two visual working memory (VWM) arrays can lead to profound interference. It is unclear, however, whether these costs arise from limitations in VWM storage capacity (Fougnie \& Marois, 2006) or from interference between the storage of one visual array and encoding or retrieval of another visual array (Cowan \& Morey, 2007). Here, we show that encoding a VWM array does not interfere with maintenance of another VWM array unless the two displays exceed maintenance capacity (Experiments 1 and 2). Moreover, manipulating the extent to which encoding and maintenance can interfere with one another had no discernable effect on dual-task performance (Experiment 2). Finally, maintenance of a VWM array was not affected by retrieval of information from another VWM array (Experiment 3). Taken together, these findings demonstrate that dual-task interference between two concurrent VWM tasks is due to a capacity-limited store that is independent from encoding and retrieval processes.
\end{abstract}

Working memory (WM) and attention are two central pillars of cognition. WM allows us to actively maintain and manipulate mental information for short periods of time (Baddeley, 1986; Baddeley \& Hitch, 1974; Logie, 1995), whereas selective attention permits the selective processing of a subset of stimuli present in the environment (Broadbent, 1958; Luck \& Vecera, 2002). In recent years, the relationship between these two cognitive constructs has become an area of intense research interest (Awh, Vogel, \& Oh, 2006; Fougnie \& Marois, 2006; Oh \& Kim, 2004; Woodman \& Luck, 2004; Woodman, Vogel, \& Luck, 2001). Such research has clearly highlighted the role of attention not only in the encoding of information into WM (Dell'Acqua \& Jolicœur, 2000; Irwin \& Gordon, 1998; Jolicœur \& Dell'Acqua, 1998, 1999; Schmidt, Vogel, Woodman, \& Luck, 2002), but also in the updating and retrieval of information from WM (Han \& Kim, 2004; Oberauer, 2002, 2003).

In contrast to WM encoding and retrieval, however, there is still little consensus on the relationship between attention and the maintenance of information in WM. Several authors have suggested that the capacity limit of WM maintenance is set by attention (Cowan, 1995, 2001, 2006; Rensink, 2000, 2002). In support of this assertion, both processes are generally considered to have a capacity limit of about three or four objects (Cowan, 2001; Pashler, 1988; Pylyshyn \& Storm, 1988; Vogel, Woodman, \& Luck, 2001). However, the similarity in capacity limits may be mere coincidence. Indeed, given that capacity for attentional tracking and WM tasks depends on various stimulus parameters such as the speed of the disks that need to be tracked (Alvarez \& Franconeri, 2007) or the complexity of the items that need to be remembered (Alvarez \& Cavanagh, 2004; but see Awh, Barton, \& Vogel, 2007), it is not difficult for capacity estimates of either attentional or WM tasks to depart from the magical number 4.

Findings demonstrating that demands on selective attention impair visual working memory (VWM) performance have also been interpreted as supporting a tight relationship between attention and WM maintenance (Awh, Jonides, \& Reuter-Lorenz, 1998; Oh \& Kim, 2004; Woodman \& Luck, 2004). However, such interference is primarily observed when the attention and VWM tasks share the same featural domain (Awh et al., 1998; Fougnie \& Marois, 2006, 2009; Oh \& Kim, 2004; Woodman \& Luck, 2004; Woodman et al., 2001), therefore suggesting that what may matter most for attention to interfere with WM is whether they operate on similar representational formats. Moreover, evidence of dual-task interference between attention and VWM does not necessarily imply that attention is solely responsible for the capacity limit of VWM. If this were the case, interference between a visuospatial attention task and a VWM task should be equivalent to the interference produced by two concurrently performed VWM tasks. This hypothesis was recently tested by introducing various secondary tasks during the retention interval of a VWM task containing a simple array of colored stimuli (Fougnie \& Marois, 2006). Not surprising, two VWM tasks strongly interfered with each other: Capacity estimates for each of the dual WM tasks were 
half those of single-task capacity. By contrast, the performance of visuospatial attention-demanding tasks during the retention interval of a VWM task generated far less interference. Although these results confirmed that attention can interfere with VWM (Awh et al., 1998; Oh \& Kim, 2004; Woodman \& Luck, 2004), they also suggested that attention is not solely responsible for the capacity limit of VWM storage.

Fougnie and Marois's (2006) finding that attention and VWM maintenance do not share the same capacity limit rests on the notion that interference during concurrent performance of two VWM tasks reveals capacity limits in VWM storage. This notion was recently challenged by Cowan and Morey, who argued that two VWM tasks may interfere with each other during encoding and retrieval, in addition to any interference observed during maintenance. If the larger dual-task costs incurred during the concurrent performance of two VWM tasks relative to the concurrent performance of an attention and a VWM task were not solely due to limits in storage capacity, this would draw into question the evidence that the capacity limit of VWM is distinct from the limitations of attentional capacity (Fougnie \& Marois, 2006). In support of their assertion, Cowan and Morey (2007) presented evidence that maintenance of one VWM array of three color stimuli interferes with the encoding of a subsequent VWM array of three colored stimuli. In their elegant procedure, participants initially encoded two sequentially presented WM displays. A postcue presented $500 \mathrm{msec}$ after the second array's offset instructed the participants which of the two visual displays to maintain in WM until probe presentation. Consequently, although Cowan and Morey's procedure required the encoding of two displays, the participants' WM was only tested for one display. Performance in the postcue condition was significantly worse than performance in a similar WM task in which only one set of three colored stimuli was presented (single-task condition). Because both the single-task and the postcue task involved maintaining and probing a single display, the lower performance in the postcue condition (postcue costs) was deemed to reflect interference between the encoding of one visual display and the maintenance of another visual display.

Cowan and Morey's (2007) findings rest on the premise that the postcue procedure specifically reveals dual-task interference resulting from encoding one VWM set while maintaining another VWM set. It is imperative to substantiate this premise, not only because of its important ramifications on the nature of the capacity limit of VWM, but also because these results conflict with prior evidence that the rate of information encoding in VWM is not affected by concurrent maintenance of another visual display (Woodman \& Vogel, 2005), suggesting that encoding and maintenance of visual features may not interfere with one another. Indeed, a closer look at Cowan and Morey's postcue procedure leaves open the possibility that postcue costs in the dual-array task may have resulted from storage limitations in WM prior to postcue presentation rather than from encoding limitations. This is because the VWM load in the dual-task postcue conditions of Cowan and Morey was six colored items, which surpasses the known capacity limits for such simple stimuli (three or four; Alvarez \& Cavanagh, 2004; Luck \& Vogel, 1997; Todd \& Marois, 2004; Vogel et al., 2001). Consequently, the participants would have had to initially store six items in order to perform the postcue task accurately, thereby placing demands on VWM storage that exceed the average participant's capacity.

Thus, one goal of the present study was to test whether postcue costs are due to WM encoding limitations, as was argued by Cowan and Morey (2007), or due to storage limitations, as was suggested above. A second goal of our study was to examine whether retrieval of information from VWM may also interfere with VWM maintenance. Although Cowan and Morey did not assess interference between VWM maintenance and retrieval, they did hypothesize that such retrieval-based interference could account for at least some of the costs observed in dual VWM tasks (Fougnie \& Marois, 2006).

To address these two goals, we carried out three experiments. We first tested the hypothesis that the postcue procedure may tax VWM maintenance rather than VWM encoding. If this hypothesis is right, postcue costs should occur when VWM storage capacity is surpassed but not when the VWM load is under capacity. Experiments 1 and 2 addressed this issue. The hypothesis also predicts that postcue costs should be the same, regardless of whether the two visual displays are presented simultaneously or sequentially, since these two display presentations have equivalent storage demands, whereas an encoding-maintenance interference account would predict greater dual-task costs in the sequential display presentation, because this presentation optimizes competition between interference and maintenance stages of VWM (Saults \& Cowan, 2007). This was tested in Experiment 2. To address the putative interference of VWM retrieval on VWM maintenance, in Experiment 3 we manipulated task response order in a dual VWM task. If retrieval of one task disrupts VWM maintenance of another, performance for the VWM task that was responded to last should be impaired relative to the VWM task that was responded to first. The results of all three experiments provide no evidence that encoding or retrieval from one VWM task interferes with maintenance of the other VWM task. Rather, it is the maintenance stage of VWM that is the source of interference in dual-task VWM conditions. We therefore conclude that interference resulting from the concurrent performance of two VWM tasks (e.g., Fougnie \& Marois, 2006) is due to competition for a limited capacity store (Luck \& Vogel, 1997; Vogel et al., 2001).

\section{EXPERIMENT 1}

If interference in the postcue task is due to storage limitations, postcue costs should only occur when the number of stimuli that are presented exceed VWM storage capacity. If interference is instead due to competition between WM encoding and maintenance processes, postcue costs would be expected for both sub- and supracapacity set sizes. To distinguish between these predictions, in Experi- 
ment 1 we presented participants with a postcue task containing either subcapacity or supracapacity VWM loads. VWM capacity for simple colored stimuli is limited to about four items (Alvarez \& Cavanagh, 2004; Vogel et al., 2001). In the subcapacity two-object postcue condition, the participants viewed two sequentially presented displays of two items. Because this condition requires storing a total of four items until presentation of the postcue, the storage limitation account predicts that postcue performance would be roughly equivalent to a two-object singletask condition, because the number of presented stimuli would generally be within the participants' capacity limit. However, in the supracapacity three-object postcue condition, which is analogous to that of Cowan and Morey (2007), greater postcue costs (relative to a three-object single-task condition) are expected because more objects (six) are presented than the typical capacity limit of four. In contrast to the storage limitation account, the encoding interference account predicts significant postcue costs in both the two-object and three-object conditions, because in both conditions, encoding of one VWM display can interfere with maintenance of another VWM display.

\section{Method}

Participants. Eighteen young adults (6 male) participated for course credit or monetary reward.

Stimuli. Colors for the circle memory set were drawn from the following list without replacement: red, brown, blue, yellow, or purple. Colors for the square memory set were drawn from a different list, also without replacement: light blue, green, pink, orange, or white. VWM stimuli measured $0.75^{\circ}$ (diameter) and were presented $3.4^{\circ}$ from fixation.

In the three-object postcue and single-task conditions, the circle stimuli appeared on the three corners of an imaginary upright triangle $\left(0^{\circ}, 120^{\circ}\right.$, and $\left.240^{\circ}\right)$, whereas the square stimuli appeared on the three corners of an inverted triangle $\left(60^{\circ}, 180^{\circ}\right.$, and $\left.300^{\circ}\right)$. In the two-object postcue and single-task conditions, the circle stimuli appeared above and below fixation and square stimuli appeared to the left and right of fixation.

Procedure. The timeline for a trial is illustrated in Figure 1. A trial began with the presentation of a central cross, which the participants were instructed to fixate. In addition, the central cross also signaled to the participants to began repeating the word the at a $2-\mathrm{Hz}$ rate for the duration of the trial. This articulatory suppression task served to minimize verbal encoding and rehearsal of the visual stimuli (Baddeley, 1986). The participants' verbalizations were monitored through speakers to confirm that they performed the articulatory suppression task.

The participants performed single-task and postcue trials intermixed within blocks. In the single-task condition, the VWM display of two or three items followed the fixation cross by $1,000 \mathrm{msec}$ and was presented for $500 \mathrm{msec}$, with equal probability that the set would contain squares or circles. Even though the postcue was not necessary for this condition, it was presented in order to equate sensory components between single-task and postcue conditions. The postcue $\left(0.75^{\circ}\right)$, which was presented $500 \mathrm{msec}$ after the offset of the VWM display, matched the shape of the VWM display stimuli and remained onscreen for 1,000 msec. After a 3,000-msec retention interval, the participants' memory was assessed using a singleprobe change detection procedure (Irwin, 1992; Vogel et al., 2001; Wheeler \& Treisman, 2002): A test display of one item matching in shape and color an item in the cued memory set. On half of the trials, the position of the tested item was the same as in the memory display. For the rest of the trials, the item was presented in one of the other locations for that shape. ${ }^{1}$ If the item had the correct color- location pairing, the participants were instructed to press a key on the keyboard labeled SAME using the index finger of the right hand. If the item had an incorrect color-location pairing, they pressed another key, labeled DIFFERENT, using the middle finger of the right hand. The participants were given as much time as they wanted to make an accurate response.

In the postcue condition, the fixation screen was followed by successive presentation of the circle and square VWM displays, with presentation order randomized. The memory displays were presented for $500 \mathrm{msec}$, with a 500 -msec offset between displays. Following this encoding period, a postcue $\left(0.75^{\circ}\right)$ that was either a black square or a black circle was shown for $1,000 \mathrm{msec}$, with the shape of the postcue (either circle or square) indicating which set to maintain in VWM. Following the 3,000-msec retention interval, the participants' color-location memory for the task-relevant display was assessed using a single-probe change detection procedure.

The task (single-task or postcue) and array size (two or three objects) conditions were randomly intermixed within two blocks of 32 self-paced trials. Before the start of the experiment, the task instructions were explained to each participant, and the participants performed 32 practice trials with performance feedback.

Analyses in this and subsequent experiments of this article were performed on accuracy data and on capacity estimates $(K)$ derived from the accuracy data (Cowan, 2001; Pashler, 1988). Because these two measures yielded statistically equivalent results, we only report the statistical tests on the accuracy data.

\section{Results and Discussion}

VWM accuracy and capacity $(K)$ for the two- or threeobject single-task and postcue conditions of Experiment 1 are shown in Figure 2. A within-subjects ANOVA was performed on the VWM accuracy performance as a function of task (single-task or postcue task) and array size (two or three). The results revealed a main effect of task $[F(1,17)=12.43, p<.005]$, with performance in the single-task condition being better than performance in the postcue condition. In addition, there was a main effect of array size $[F(1,17)=6.55, p<.05]$, with higher performance for two-object displays. Most important, there was an interaction between task and array size $[F(1,17)=$ 39.03, $p<.0001]$. Planned comparisons using paired $t$ tests examined the difference between postcue and single-task VWM performance within both the two- and the three-object conditions. Although VWM performance revealed large postcue costs when the VWM displays contained three stimuli $[t(17)=3.78, p<.005]$, there was no evidence of such costs with displays of two items $[t(17)=$ $0.46, p=.65]$. This suggests that postcue costs occur only for VWM loads that exceed estimates of VWM storage capacity. These results are consistent with the storagelimit account of postcue costs but not with the encoding interference account.

It is conceivable, however, that encoding costs vary with WM set size. That is, an increase in the number of objects stored in WM of Task 1 may accentuate the potential for interference with WM encoding of Task 2. If so, the results of Experiment 1 might not strictly rule out the encoding interference account. However, a critical difference between this account and the storage-limit account is that the latter predicts that the increase in postcue costs in the three-object condition relative to the two-object condition should be related to an individual participant's single-task capacity, such that the participants with poorer single-task 


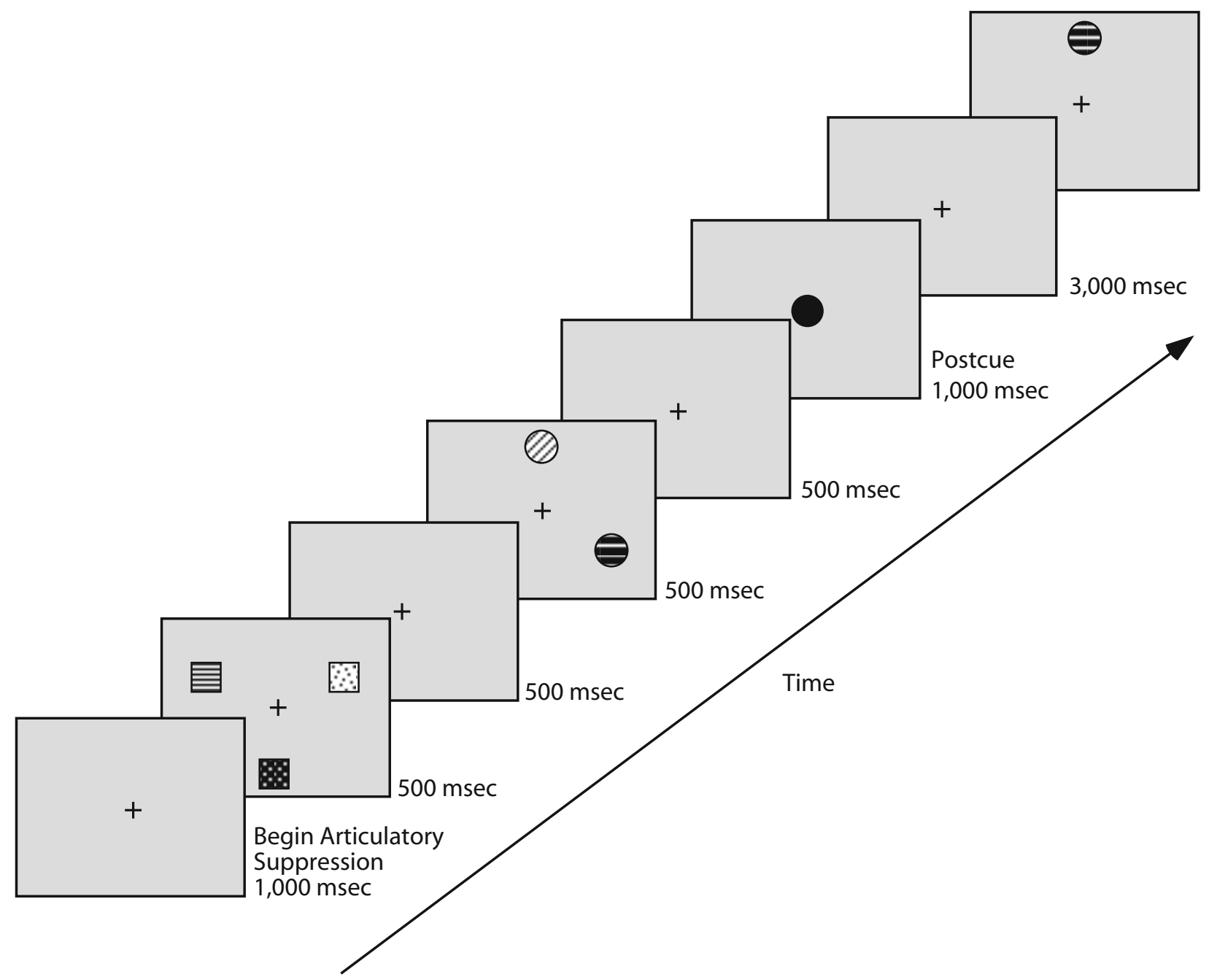

Figure 1. Trial timeline for the three-object postcue condition of Experiment 1. Different fill patterns represent different solid colors. A trial began with the presentation of a central fixation cross that informed the participants to begin the articulatory suppression task. The order of the VWM sets (circle or square first) was randomly selected for each trial. The trial timeline was identical in the singletask condition, except that the participants were shown only one VWM set (selected at random).

performance will show higher postcue costs. By contrast, interference between encoding and maintenance would predict, if anything, the reverse pattern - that the participants with higher WM capacity would show greater postcue costs, since they have more objects stored in VWM during the subsequent encoding phase.

A correlational analysis examining the relationship between an individual's performance in the three-object single-task condition (a measure of VWM capacity) and the difference in accuracy between the three-object postcue condition and the two-object postcue condition (a measure of postcue costs at high WM loads) revealed a strong negative correlation $(r=-.47, p<.05)$ such that the participants with low single-task WM performance had increased costs in the three-object condition (Figure 3). ${ }^{2}$ The finding that the participants with lower VWM capacity showed larger postcue costs is a direct prediction of our account and is not consistent with the possibility that this decrease is due to interference between encoding and maintenance.

\section{EXPERIMENT 2}

The results of Experiment 1 suggest that supracapacity loads are a necessary precondition for observing postcue costs. The goal of Experiment 2 was to replicate these results and provide converging evidence that postcue costs are better explained by storage limitations than by interference between encoding of one VWM display and storage of another VWM display. The storage limit account implies that dual-task impairment is due to interference between two visual arrays for retention in a capacitylimited store. As such, this model predicts no role for the encoding phase of VWM in accounting for dual-task limitations. In contrast, because the encoding interference account posits that dual-task impairment results from a 


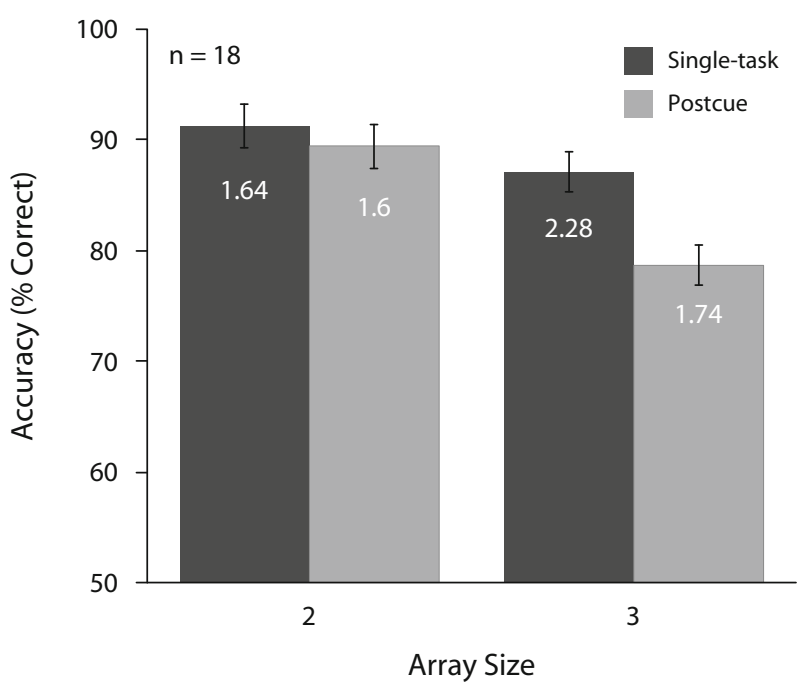

Figure 2.VWM accuracy results for the single-task and postcue conditions of Experiment 1 as a function of array size. Error bars represent within-subjects errors of the mean. Capacity $(K)$ for each condition is listed on that condition's bar.

conflict between encoding of a VWM array and retention of another array (Cowan \& Morey, 2007), dual-task costs should be sensitive to the overlap between the encoding and retention phases of each VWM array. Specifically, Saults and Cowan (2007) argued that simultaneous presentation of two WM stimulus sets reduces the potential for interference between the encoding of one set and the storage of another set, because both stimulus sets are encoded concurrently. In Experiment 2, we tested how postcue costs are affected by the overlap between encoding and retention by presenting the stimulus displays for the two VWM tasks either sequentially (SEQ condition) or simultaneously (SIM condition). In this experiment, participants performed blocks of single-task trials or postcue trials. In the postcue blocks, the participants memorized a set of two or three colored squares and a set of two or three colored circles, with the sets being presented either simultaneously or sequentially. As in Experiment 1, after the offset of the last display, a postcue indicated which stimulus set would be tested. A storage limit account predicts that SIM and SEQ presentations will lead to equivalent performance, because the two conditions place equivalent demands on storage capacity. However, because the encoding interference account postulates that the conflict in dual VWM tasks is between encoding of one set and maintenance of another, this account predicts worse performance in the SEQ presentation and that this cost for sequentially presented stimuli will be greater for the three-object condition. In addition, as in Experiment 1, we predicted that performance in the postcue condition would be worse than single-task performance for the three-object but not the two-object condition.

\section{Method}

Participants. Sixteen young adults $(7$ male) participated for course credit or monetary reward.
Stimuli. The stimuli were identical to those of Experiment 1.

Procedure. The participants completed two single-task blocks of 16 trials and two postcue blocks of 32 trials. The blocks were presented in alternating order, with the starting block (single-task or postcue) counterbalanced across participants. Prior to the study, the participants performed 8 single-task and 16 postcue practice trials. We blocked single-task and postcue trials, as opposed to intermixing them, to avoid confusion between the single-task and postcue SEQ trials, since these two trial types were identical for the first $1,000 \mathrm{msec}$ of a trial.

Single-task trials were similar to those in Experiment 1, except that the retention interval was increased from 3,000 to 4,000 msec in order to provide a more conservative baseline to evaluate postcue costs. In Experiment 1, the retention interval for the single task trials, measured from the end of the encoding interval, corresponded to the retention interval of the second array in the postcue trials $(3,000 \mathrm{msec})$. However, because the first array in these postcue trials had a retention interval that was $1,000 \mathrm{msec}$ longer, it is possible that the overall longer retention intervals for the postcue trials $(4,000$ and 3,000 msec) than for the single task trials $(3,000 \mathrm{msec})$ may have inflated our estimate of postcue costs. Supporting this possibility, change detection accuracy for three-object postcue trials was 3\% lower for the first than for the second array (there was no difference for two-object postcue trials). Although this difference in postcue performance for the first and second arrays is too small to explain the postcue costs $(>10 \%)$ observed in Experiment 1, this problem was alleviated in Experiment 2 by using the longest of the two postcue retention intervals as the retention interval for the single-task trials.

The sequentially presented postcue trials were identical to the postcue trials of Experiment 1. In the SIM condition, the three-circle and three-square memory sets were presented simultaneously for $1,000 \mathrm{msec}$, followed by a 1,000 -msec gap between stimulus presentation and postcue onset, which in turn was followed by a retention interval of $3,000 \mathrm{msec}$. The total encoding period was equivalent $(2 \mathrm{sec})$ across the SIM and SEQ conditions. The two-object and three-object trials were intermixed within blocks.

\section{Results and Discussion}

VWM accuracy and capacity for the single-task SIM and SEQ conditions are shown in Figure 4.

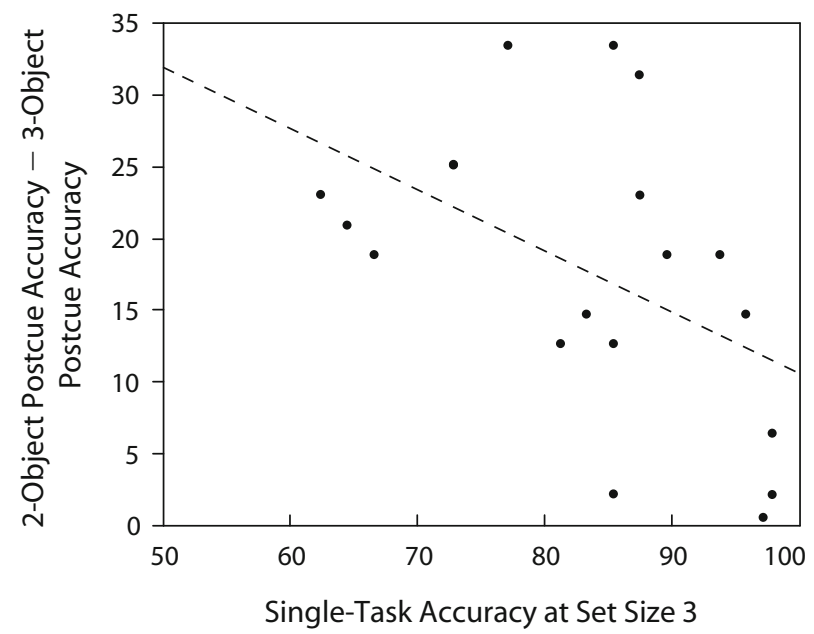

Figure 3. Scatterplot showing the relationship of participant's single-task accuracy (percentage correct) for three-object trials $(x$-axis) and the subtraction of three-object postcue accuracy from two-object postcue accuracy ( $y$-axis) for Experiment 1. The linear trend $(r=-.47$; dotted line) was significant $(p<.05)$. 
In Experiment 1, we found significant postcue costs in the three-object but not in the two-object condition. To examine postcue performance relative to single-task performance in the present study, single-task and postcue trials were entered into a $2 \times 3$ ANOVA with the factors of array size (two or three) and task (single task, SIM, or SEQ). There was a main effect of array size such that WM performance was better in the two-object than in the threeobject condition $[F(1,15)=14.11, p<.005]$. There was a main effect of task $[F(2,30)=4, p<.05]$, with single-task performance being better than that in either the SEQ or the SIM condition. We also observed an interaction between task and array size $[F(2,30)=3.63, p<.05]$. As can be seen in Figure 4, the interaction appears to be driven by large postcue costs in the three-object condition, but not in the two-object condition, consistent with the results of Experiment 1. Indeed, separate one-way ANOVAs were conducted on the two-object and three-object conditions, examining the effect of task (single task, SIM, or SEQ). In the two-object condition, there was no effect of task $[F(2,30)=0.86, p=.43]$, but there was an effect in the three-object condition $[F(2,30)=5.63, p<.05]$. Thus, we replicated the main finding of Experiment 1, which is that significant postcue costs are only observed for VWM loads that exceed estimates of VWM storage capacity. Unlike in Experiment 1, however, we could not perform the correlational analysis to test whether the participants' VWM performance in the three-object single-task condition was correlated with the magnitude of the array size effect in the postcue conditions because of insufficient

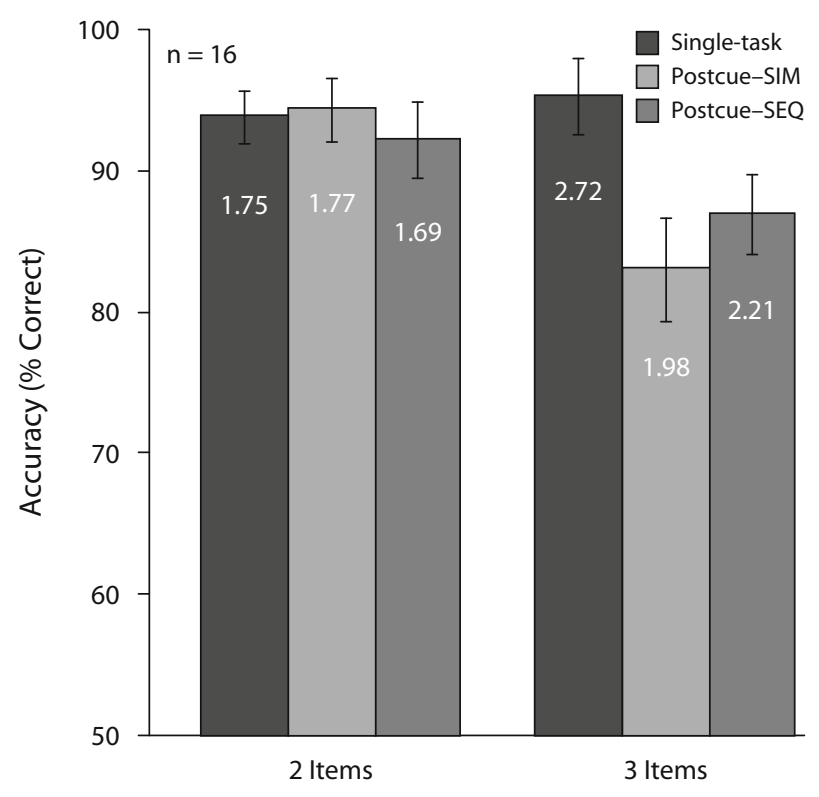

Array Size

Figure 4. VWM accuracy results for the single-task simultaneous (SIM) and sequential (SEQ) conditions of Experiment 2 as a function of array size. Error bars represent within-subjects errors of the mean. Capacity $(K)$ for each condition is listed on that condition's bar. variability (only 6 of the 16 participants made an error during the three-object single-task condition).

To examine the effect of stimulus presentation order (SIM vs. SEQ), VWM accuracy for the postcue trials was entered into a $2 \times 2$ ANOVA with the factors of array size (2 or 3 ) and presentation (SIM or SEQ). There was a main effect of array size, such that WM performance was better in the two-object than in the three-object condition $[F(1,15)=14.76, p<.005]$. There was no main effect of presentation $[F(1,15)=0.01, p=.92]$ and no interaction between presentation and array size $[F(1,15)=2.23, p=$ .16]. Because performance in the SIM condition and that in the SEQ condition were indistinguishable, the results provide no evidence that manipulating the overlap between the encoding stage of one VWM task and the maintenance stage of another VWM task affected dual-task interference. These results are inconsistent with Saults and Cowan's (2007) prediction that the simultaneous presentation of two WM arrays should ameliorate dual-task performance relative to sequential presentation, because it would eliminate potential interference between WM encoding and maintenance.

The results of the SEQ/SIM manipulation rest on the assumption that the participants performed the tasks as instructed. Specifically, equivalent postcue costs for the SIM and SEQ presentations would also be expected if the participants disregarded the experimenter's instructions and encoded the stimulus sets sequentially even during simultaneous presentation. However, this account implies that the participants would have encoded the VWM stimuli in the SIM condition using a suboptimal strategy, because, according to Saults and Cowan (2007), this strategy would promote interference between encoding of a VWM array and maintenance of another. In the absence of any compelling reason for the participants to select this nonadvantageous strategy, the more parsimonious explanation for our results is that encoding and maintenance do not mutually interfere.

\section{EXPERIMENT 3}

The first two experiments cast doubt on the assertion that interference in VWM dual-task experiments results from conflict between the encoding and maintenance phases of VWM. However, it has also been suggested that VWM dual-task costs may be exaggerated by interference from the retrieval of one VWM display with the maintenance of a second display (Cowan \& Morey, 2007). If engaging in retrieval for one VWM display impairs the maintenance of another VWM display in a dual-task setting, the performance of a VWM task will be affected by whether that task is preceded by one that involves VWM retrieval. Thus, one method of determining the impact of VWM retrieval on VWM maintenance is to manipulate the order of responses in a dual VWM task and to determine whether VWM performance for the second response is more impaired than for the first response. Since in our previous dual VWM experiment (Fougnie \& Marois, 2006, Experiment 2), we did not manipulate response order, here 
we replicate that study using the same paradigm while varying the order of the probe displays.

\section{Method}

Participants. Fifteen young adults (6 male) participated for monetary reward.

Stimuli. For each trial, the two color sets (set one: light blue, dark green, red, white, and purple; set two: light green, brown, pink, blue, and yellow) were randomly assigned to one of the two VWM tasks (VWM1 or VWM2). The three VWM1 stimuli were circles $\left(0.78^{\circ}\right)$ that could appear at any of the four locations $3.3^{\circ}$ from fixation on the horizontal and vertical axes, whereas the VWM2 stimuli were squares that could appear at the corresponding locations of the two $45^{\circ}$ diagonal axes.

Procedure. The experimental procedure was similar to that of the single- and dual-VWM tasks used in Fougnie and Marois (2006). The participants performed an articulatory suppression task, which consisted of repeating the word the at a $2-\mathrm{Hz}$ rate, beginning $1,000 \mathrm{msec}$ prior to each trial and ending only after the responses for both tasks had been collected. A trial began with the presentation of a VWM1 display of zero or three stimuli for $400 \mathrm{msec}$. Following a 1,200msec encoding interval, the VWM2 display $(0,1$, or 3 stimuli) was presented for $400 \mathrm{msec}$. After a 9,000-msec retention interval, the two probe displays were presented in a randomized order. Probe displays contained a single colored shape (circle for VWM1 and square for VWM2), and the participants had to indicate whether this probe shape matched the color and location of one of the memory display items (50\% match trials). Nonmatching VWM1 probes involved an incorrect pairing of color and location. Nonmatching VWM2 probes differed from those in VWM1, since we could not switch color and location when there was only one VWM2 item. For trials with a VWM2 array size of one, nonmatching VWM2 probes entailed a new color or a new location (with equal probability), whereas for trials with a VWM2 array size of three, nonmatching VWM2 probes entailed a new color, a new location, or an incorrect pairing of color and location (with equal probability). For the VWM1 probe, the participants made an unspeeded response by pressing one of two keys with the index and middle fingers of their left hand to indicate whether the probe item matched one of the VWM1 stimuli. A similar response was made for the VWM2 probe with the two corresponding fingers of the right hand. To control for sensory and motor demands across conditions, single-task trials still involved the presentation of two visual displays and required two manual responses. In those trials, the stimuli for one of the two memory arrays were replaced by four black dummy triangles $\left(0.78^{\circ}\right)$ appearing at the four potential stimulus locations (this change occurred for VWM1 on $25 \%$ of all trials and for VWM2 on $33 \%$ of all trials). In addition, the VWM probe display for the dummy memory set was replaced by a screen with a number sign (\#) above fixation, indicating that the participants should simply press the space bar.

The participants completed one practice block and four experimental blocks of 48 trials each. The experimental conditions were randomly intermixed within blocks.

\section{Results and Discussion}

Accuracy for the VWM1 and VWM2 tasks is shown in Figures $5 \mathrm{~A}$ and $5 \mathrm{~B}$, whereas Tables $1 \mathrm{~A}$ and $1 \mathrm{~B}$ list the capacity estimates $(K)$ for each condition. Accuracy on the VWM1 task (Figure 5A) was analyzed in a 2 (response order) $\times 3$ (VWM2 array size) within-subjects ANOVA. There was a main effect of VWM2 array size $[F(2,28)=21.69, p<.0001]$, but no effect of response order $[F(1,14)=2.1, p=.18]$, and no interaction between VWM2 array size and response order $[F(2,28)=0.56$, $p>.5]$. VWM2 accuracy (Figure 5B) was analyzed in a 2 (response order $) \times 2($ VWM 2 array size $) \times 2($ VWM1
Table 1A

Capacity Estimates (K; Cowan, 2001) for the VWM1 Task of Experiment 3 As a Function of Task Array Size and Response Order

\begin{tabular}{|c|c|c|c|c|}
\hline & & \multicolumn{3}{|c|}{ VWM1 Capacity } \\
\hline & & Single-Task & 1VWM2 & 3VWM2 \\
\hline 3VWM1 & Probed 1st & 1.92 & $\begin{array}{l}2.04 \\
178\end{array}$ & 1.22 \\
\hline
\end{tabular}

Note-1VWM2, Task 2 VWM with array size 1; 3VWM2, Task 2 VWM with array size 3; 3VWM1, Task1 VWM with array size 3 .

Table 1B

Capacity Estimates (K; Cowan, 2001) for the VWM2 Task of Experiment 3 As a Function of Task Array Size and Response Order

\begin{tabular}{llcc}
\hline & & \multicolumn{2}{c}{ VWM2 Capacity } \\
\cline { 3 - 4 } & & Single-Task & 3VWM1 \\
\hline \multirow{2}{*}{ 1VWM2 } & Probed 1st & 0.94 & 0.74 \\
& Probed 2nd & 0.92 & 0.74 \\
3VWM2 & Probed 1st & 2.29 & 2.04 \\
& Probed 2nd & 2.29 & 1.15 \\
\hline
\end{tabular}

Note-3VWM1, Task 1 VWM with array size 3; 1VWM2, Task 2 VWM with array size $1 ; 3 \mathrm{VWM} 2$, Task $2 \mathrm{VWM}$ with array size 3 .

array size) ANOVA. This analysis revealed main effects of both VWM1 array size $[F(1,14)=27.24, p<.001]$ and VWM2 array size $[F(1,14)=33.12, p<.0001]$ but no interaction between these two factors $(p>.1)$. Most important, there was no main effect of response order $[F(1,14)=1.13, p=.31]$, and response order did not interact with any of the other factors (all $p \mathrm{~s}>.15$ ). These results replicate the findings of Fougnie and Marois (2006) in demonstrating that concurrent maintenance of two VWM loads causes interferences between them. More important, the results indicate that this dual-task cost is not due to retrieval of one VWM array interfering with maintenance of another, because response order did not affect VWM performance.

Although the results of Experiment 3 support the hypothesis that the dual-task costs observed by Fougnie and Marois (2006) were not due to interference at retrieval, there is an alternative account that is consistent with these results. According to this account, the lack of a response order effect does not indicate that retrieval does not affect maintenance, but, rather, indicates that retrieval of one VWM array and maintenance of another mutually and symmetrically interfere with each other. That is to say, if retrieval of one VWM array interferes with maintenance of another VWM array to the same extent that maintenance of that second VWM interferes with retrieval of that first VWM array, these reciprocal interference costs would yield similar task performance regardless of response order. There are two reasons, however, to suggest that this alternative account is unlikely to be viable. First, it requires that the reciprocal interference between retrieval and maintenance be equal, yet there is no a priori reason to suggest that such interference should be evenly matched. Second, and most important, there is considerable evidence to suggest that there is an asymmetric pattern of interference between retrieval and secondary task demands in dual-task settings. Specifically, retrieval 


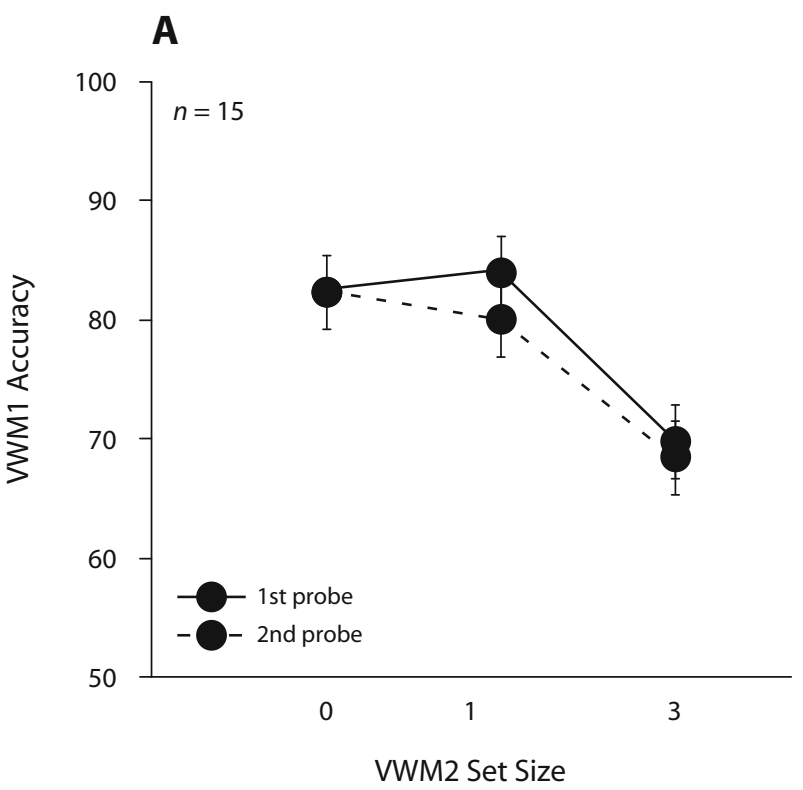

B

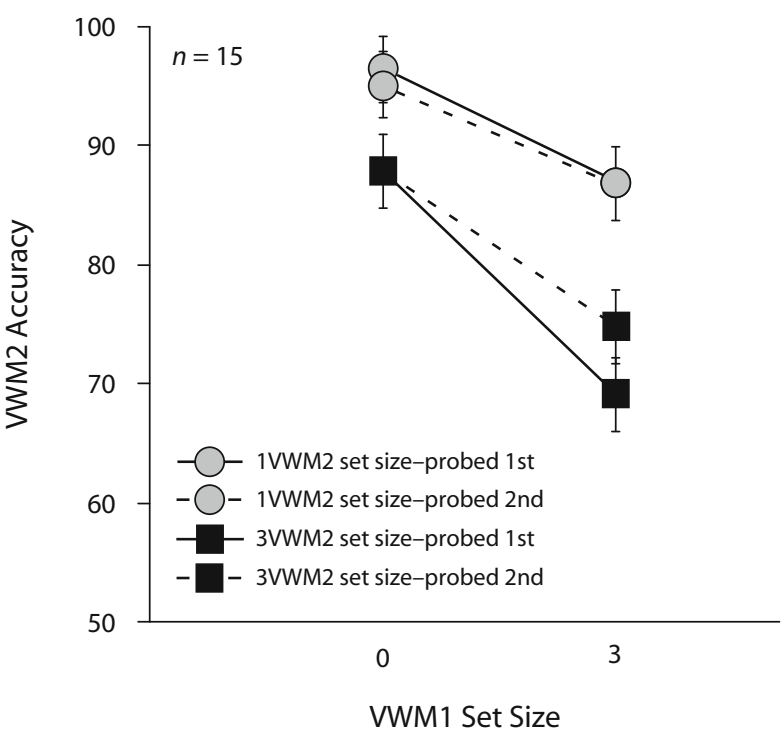

Figure 5. VWM accuracy results for the VWM1 (A) and VWM2 (B) tasks of Experiment 3 as a function of probe order and array size for the VWM tasks. Error bars represent withinsubjects errors of the mean.

efficacy is rarely affected by the concurrent performance of a continuously demanding secondary task, but retrieval may significantly impair that secondary task's performance (Craik, Govoni, Naveh-Benjamin, \& Anderson, 1996; Kellogg, Cocklin, \& Bourne, 1982; Naveh-Benjamin, Craik, Perretta, \& Tonev, 2000; Naveh-Benjamin, Kilb, \& Fisher, 2006). Thus, retrieval from WM appears to be an obligatory, protected process that is unimpaired by concurrent task demands (Craik et al., 1996; Naveh-Benjamin, Craik, Guez, \& Dori, 1998; Naveh-Benjamin et al., 2000; NavehBenjamin et al., 2006).
On the basis of the evidence discussed above, we conclude that the process of retrieving information from one VWM task does not interfere with the maintenance of information of another VWM task. Of course, our results do not rule out the existence of retrieval-based interference: Such interference might be revealed, for example, if the WM paradigms made greater demands on attention and/or executive processing. Rather, what the present results clearly suggest is that such retrieval-based interference, if it exists, can hardly account for the considerable dual-VWM costs observed in the present experiment or in those of Fougnie and Marois (2006), because such costs can be observed independently of any potential retrievalbased interference.

In summary, the results of Experiment 3, together with those of Experiments 1 and 2, are consistent with the hypothesis that the main source of dual VWM costs is interference between the maintenances of two VWM arrays rather than between maintenance of one array and encoding or retrieval of another array.

\section{GENERAL DISCUSSION}

Using a dual-task experimental approach, Fougnie and Marois (2006) showed that the capacity limit of VWM maintenance is not solely based on a unitary attentional mechanism, but is instead a product of visuospatial, central amodal, and content-specific sources of information processing. Their findings rest on the assumption that concurrent performance on two VWM tasks is primarily constrained by the capacity-limited stage of maintaining information in VWM. However, dual-task interference can theoretically occur at any processing stage (Cowan \& Morey, 2007; Pashler, 1994). Indeed, it has been argued that dual-task costs during the performance of two WM tasks may not necessarily result from competition during WM maintenance, since such costs could also arise from interference between encoding or retrieval of one WM task and maintenance of another (Cowan \& Morey, 2007).

To demonstrate the existence of encoding costs in dual VWM tasks, Cowan and Morey (2007) developed a postcue procedure that involves the encoding of two sets of WM stimuli but the retention of only one of these two stimulus sets for the entire retention interval. When compared with a standard single-task procedure, postcue performance was impaired only when the two sets of WM stimuli shared a modality (visual or verbal). These modality-specific postcue costs were argued to reflect interference between encoding information in one task and maintaining information in the second task (Cowan $\&$ Morey, 2007). However, the postcue procedure does not eliminate demands on maintenance, because the participants still have to store two sets of WM stimuli for several hundred milliseconds before discarding one set. Thus, postcue costs could occur because of encoding costs and/or storage capacity limitations. Moreover, such storage costs would be especially important in limiting postcue performance when the procedure requires participants to store an amount of VWM stimuli (6 items) that exceeds 
estimates of VWM capacity (three or four items; Vogel et al., 2001), as it did in Cowan and Morey's study.

In three experiments, we investigated the role of encoding, storage, and retrieval limitations in dual-VWM tasks and found that postcue costs are better accounted for by interference between the maintenance phases of two WM tasks rather than between encoding or retrieval of one task and maintenance of another. Specifically, in Experiment 1, we manipulated the number of items in the postcue procedure and observed postcue costs only when the number of stimuli exceeded estimates of VWM storage capacity, a result that is inconsistent with an encoding interference account of dual VWM postcue costs. Experiment 2 provided further evidence that postcue costs do not arise from interference between encoding of one VWM array and maintenance of another VWM array, because such costs were unaffected by a manipulation that affected the extent to which the encoding stage of one VWM array overlapped with the encoding stage of another VWM array. Finally, in Experiment 3, we used a response order manipulation in a dual VWM task to demonstrate that the retrieval of information from one VWM array does not impair retention of a second VWM array, thereby arguing against the possibility that costs arising from the concurrent performance of two VWM tasks result from interference between retrieval and maintenance processes. Taken together, these results are consistent with Fougnie and Marois's (2006) finding that dual-task costs in VWM arise from interference between the maintenance requirements of two object arrays in VWM. Our results are also highly consistent with Woodman and Vogel's (2005) demonstration that a VWM storage load does not affect the rate of stimulus encoding for another VWM task.

Why does maintaining information in VWM not interfere with encoding or retrieval? One possibility is that although encoding and retrieval are controlled processes closely linked to attention (Cantor \& Engle, 1993), maintenance in VWM could be supported by processes relatively isolated from attentional demands. Indeed, although it is often proposed that attention is critical for maintaining representations in VWM, there is very little evidence of interference between visuospatial attention and nonspatial, object WM maintenance (Awh \& Jonides, 2001; Awh et al., 1998; Woodman et al., 2001). Dual-task interference between attention and VWM maintenance seems to occur only when spatial information is relevant for both tasks (Awh \& Jonides, 2001; Awh et al., 1998; Oh \& Kim, 2004; Woodman \& Luck, 2004). For example, shifts of visuospatial attention during a VWM retention interval interfere with memory for spatial location, but not for object identity (Awh \& Jonides, 2001; Awh et al., 1998). It has been proposed that such selective VWM-attention interference may occur because shifts of visuospatial attention are used to rehearse spatial locations but not object identity during a VWM retention interval (Awh \& Jonides, 2001; Awh et al., 1998; Awh et al., 2006; Postle, Awh, Jonides, Smith, \& D'Esposito, 2004; Smith \& Jonides, 1998; Smyth \& Scholey, 1994). However, since dual-task costs between attention and VWM occur only when both tasks utilize spatial information, these costs might reflect not competition for shared attentional resources, but rather interference (crosstalk) between attention and WM for access to a common representational format (e.g., space) (Dutta, Schweickert, Choi, \& Proctor, 1995; Navon, 1984; Navon \& Miller, 1987).

In contrast to its conditional involvement in VWM maintenance, attention appears to play a ubiquitous role in encoding and retrieving information from VWM. Strong dual-task costs arise whenever attentional demands occur concomitant with WM encoding (Dell'Acqua \& Jolicœur, 2000; Irwin \& Gordon, 1998; Jolicœur, 1999; Jolicœur \& Dell'Acqua, 1998, 1999), or with the retrieval and manipulation of WM contents (Garavan, 1998; Han \& Kim, 2004; Oberauer, 2002, 2003), even when the tasks differ in modality. Thus, although dual-task costs between VWM maintenance and attention only occur if there is overlap in the task-relevant information, it appears that attention plays a general role in VWM encoding and retrieval processes. This may suggest that VWM encoding and retrieval depend on attentional processes not necessary for the continuous maintenance of information in VWM. This suggestion does not imply that VWM maintenance is completely independent of attentional resources, for attention could be transiently involved in maintenance (Schneider, 1999) - perhaps serving only to refresh the mnemonic material (Awh \& Jonides, 2001), thereby allowing attentional demands to be efficiently split between encoding of a VWM task and maintenance of another. Clearly, future research is necessary in order to determine whether attention is the key cognitive component that can account for the independence of VWM maintenance from encoding and retrieval.

\section{CONCLUSION}

Experimenters have often used a dual-task methodology to determine whether WM contains separate modalityspecific stores (Baddeley 1986; Cocchini, Logie, Della Sala, MacPherson, \& Baddeley, 2002; Cowan \& Morey, 2007; Fougnie \& Marois, 2006; Morey \& Cowan, 2005) and to examine the relationship between WM and attention (Awh et al., 1998; Fougnie \& Marois, 2006; Irwin \& Gordon, 1998; Oh \& Kim, 2004; Woodman \& Luck, 2004; Woodman et al., 2001). When the source of dual-task costs can be identified, this method can provide a powerful demonstration of the extent to which two tasks share common processes (Pashler, 1994). In a previous study (Fougnie \& Marois, 2006), we found that the pairing of two VWM tasks produced significantly more dual-task costs than the pairing of a verbal WM task and a VWM task or an attentional tracking and VWM task, suggesting that there was a capacity-limited process shared by the two VWM tasks that is not shared by the other tasks. But, as was described by Cowan and Morey (2007), such dual VWM costs could, in principle, occur at any stage of VWM processing. By demonstrating that neither encoding nor retrieval interfere with VWM maintenance, the present study allows us to conclude that when two VWM tasks compete for limited resources, the resultant interference occurs primarily during maintenance, rather than during encoding or retrieval. 


\section{AUTHOR NOTE}

We thank Olivia Cheung, Stephenie Harrison, Roy Luria, Susan Courtney, and Bradley Gibson for helpful comments on an earlier version of the manuscript. This work was supported by NIMH Grant R01MH70776 to R.M. Correspondence concerning this article should be addressed to D. Fougnie, Department of Psychology, Vanderbilt University, 627 Wilson Hall, 111 21st Ave S., Nashville, TN 37203 (e-mail: d.fougnie@vanderbilt.edu).

\section{REFERENCES}

Alvarez, G. A., \& Cavanagh, P. (2004). The capacity of visual shortterm memory is set both by visual information load and by number of objects. Psychological Science, 15, 106-111.

Alvarez, G. A., \& Franconeri, S. L. (2007). How many objects can you attentively track? Evidence for a resource-limited tracking mechanism. Journal of Vision, 7(13, Art. 14), 1-10.

Awh, E., Barton, B., \& Vogel, E. K. (2007). Visual working memory represents a fixed number of items regardless of complexity. Psychological Science, 18, 622-628.

AwH, E., \& Jonides, J. (2001). Overlapping mechanisms of attention and spatial working memory. Trends in Cognitive Sciences, $\mathbf{5}$, 119-126.

Awh, E., Jonides, J., \& Reuter-Lorenz, P. A. (1998). Rehearsal in spatial working memory. Journal of Experimental Psychology: Human Perception \& Performance, 24, 780-790.

Awh, E., Vogel, E. K., \& OH, S. H. (2006). Interactions between attention and working memory. Neuroscience, 139, 201-208.

Baddeley, A. D. (1986). Working memory. New York: Oxford University Press.

BadDeley, A. D., \& Hitch, D. J. (1974). Working memory. In G. H. Bower (Ed.), The psychology of learning and motivation: Advances in research and theory (Vol. 8, pp. 47-89). New York: Academic Press.

Broadbent, D. E. (1958). Perception and communication. London: Pergamon.

CANTOR, J., \& EngLE, R. W. (1993). Working-memory capacity as longterm memory activation: An individual-differences approach. Journal of Experimental Psychology: Learning, Memory, \& Cognition, 19, 1101-1114.

Cocchini, G., Logie, R. H., Della Sala, S., MacPherson, S. E., \& BADDELEY, A. D. (2002). Concurrent performance of two memory tasks: Evidence for domain-specific working memory systems. Memory \& Cognition, 30, 1086-1095.

Cowan, N. (1995). Attention and memory: An integrated framework. New York: Oxford University Press.

Cowan, N. (2001). The magical number 4 in short-term memory: A reconsideration of mental storage capacity. Behavioral \& Brain Sciences, 24, 87-114.

Cowan, N. (2006). Working memory capacity. New York: Psychology Press.

Cowan, N., \& Morey, C. C. (2007). How can dual-task working memory retention limits be investigated? Psychological Science, 18, 686-688.

Craik, F. I., Govoni, R., Naveh-Benjamin, M., \& Anderson, N. D. (1996). The effects of divided attention on encoding and retrieval processes in human memory. Journal of Experimental Psychology: General, 125, 159-180.

Dell'AcQua, R., \& Jolicceur, P. (2000). Visual encoding of patterns is subject to dual-task interference. Memory \& Cognition, 28, 184-191.

Dutta, A., Schweickert, R., Choi, S., \& Proctor, R. W. (1995). Cross-task cross talk in memory and perception. Acta Psychologica, 90, 49-62.

Fougnie, D., \& MARoIs, R. (2006). Distinct capacity limits for attention and working memory: Evidence from attentive tracking and visual working memory paradigms. Psychological Science, 17, 526-534.

Fougnie, D., \& MaroIs, R. (2009). Attentive tracking disrupts feature binding in visual working memory. Visual Cognition, 17, 48-66.

GaraVAn, H. (1998). Serial attention within working memory. Memory \& Cognition, 26, 263-276.

HaN, S. H., \& KIM, M. S. (2004). Visual search does not remain efficient when executive working memory is working. Psychological Science, 15, 623-628.

IRWIN, D. E. (1992). Memory for position and identity across eye move- ments. Journal of Experimental Psychology: Learning, Memory, \& Cognition, 18, 307-317.

Irwin, D. E., \& Gordon, R. D. (1998). Eye movements, attention and trans-saccadic memory. Visual Cognition, 5, 127-155.

JoLICEUR, P. (1999). Dual-task interference and visual encoding. Journal of Experimental Psychology: Human Perception \& Performance, 25, 596-616.

Joliceur, P., \& Dell'Acqua, R. (1998). The demonstration of shortterm consolidation. Cognitive Psychology, 36, 138-202.

Jolicceur, P., \& Dell'AcQua, R. (1999). Attentional and structural constraints on visual encoding. Psychological Research, 62, 154-164.

Kellogg, R. T., Cocklin, T., \& Bourne, L. E., JR. (1982). Conscious attentional demands of encoding and retrieval from long-term memory. American Journal of Psychology, 95, 183-198.

LogIE, R. H. (1995). Visuo-spatial working memory. Hillsdale, NJ: Erlbaum.

LuCK, S. J., \& Vecera, S. P. (2002). Attention: From tasks to mechanisms. In S. Yantis (Ed.), Stevens 'handbook of experimental psychology (Vol. 1, pp. 235-286). New York: Wiley.

LucK, S. J., \& Vogel, E. K. (1997). The capacity of visual working memory for features and conjunctions. Nature, 390, 279-281.

Morey, C. C., \& Cowan, N. (2005). When do visual and verbal memories conflict? The importance of working-memory load and retrieval. Journal of Experimental Psychology: Learning, Memory, \& Cognition, 31, 703-713.

Naveh-Benjamin, M., Craik, F. I. M., Guez, J., \& Dori, H. (1998). Effects of divided attention on encoding and retrieval processes in human memory: Further support for an asymmetry. Journal of Experimental Psychology: Learning, Memory, \& Cognition, 24, 1091-1104.

Naveh-Benjamin, M., Craik, F. I. M., Perretta, J. G., \& Tonev, S. T. (2000). The effects of divided attention on encoding and retrieval processes: The resiliency of retrieval processes. Quarterly Journal of Experimental Psychology, 53A, 609-625.

Naveh-Benjamin, M., Kilb, A., \& Fisher, T. (2006). Concurrent task effects on memory encoding and retrieval: Further support for an asymmetry. Memory \& Cognition, 34, 90-101.

Navon, D. (1984). Resources-A theoretical soup stone? Psychological Review, 91, 216-234.

Navon, D., \& Miller, J. (1987). Role of outcome conflict in dual-task interference. Journal of Experimental Psychology: Human Perception \& Performance, 13, 435-448.

Oberauer, K. (2002). Access to information in working memory: Exploring the focus of attention. Journal of Experimental Psychology: Learning, Memory, \& Cognition, 28, 411-421.

Oberauer, K. (2003). Selective attention to elements in working memory. Experimental Psychology, 50, 257-269.

Он, S.-H., \& Kıм, M.-S. (2004). The role of spatial working memory in visual search efficiency. Psychonomic Bulletin \& Review, 11, 275-281

Pashler, H. (1988). Familiarity and visual change detection. Perception \& Psychophysics, 44, 369-378.

Pashler, H. (1994). Dual-task interference in simple tasks: Data and theory. Psychological Bulletin, 116, 220-244.

Postle, B. R., Awh, E., Jonides, J., Smith, E. E., \& D'Esposito, M. (2004). The where and how of attention-based rehearsal in spatial working memory. Cognitive Brain Research, 20, 194-205.

Pylyshyn, Z. W., \& STORM, R. W. (1988). Tracking multiple independent targets: Evidence for a parallel tracking mechanism. Spatial Vision, 3, 179-197.

Rensink, R. A. (2000). The dynamic representation of scenes. Visual Cognition, 7, 17-42.

Rensink, R. A. (2002). Change detection. Annual Review of Psychology, 53, 245-277.

Saults, J. S., \& Cowan, N. (2007). A central capacity limit to the simultaneous storage of visual and auditory arrays in working memory. Journal of Experimental Psychology: General, 136, 663-684.

Schmidt, B. K., Vogel, E. K., Woodman, G. F., \& Luck, S. J. (2002). Voluntary and automatic attentional control of visual working memory. Perception \& Psychophysics, 64, 754-763.

SCHNEIDER, W. X. (1999). Visual-spatial working memory, attention, and scene representation: A neuro-cognitive theory. Psychological Research, 62, 220-236.

SMith, E. E., \& JonidES, J. (1998). Neuroimaging analyses of human 
working memory. Proceedings of the National Academy of Sciences, 95, 12061-12068

SMYTH, M. M., \& Scholey, K. A. (1994). Interference in immediate spatial memory. Memory \& Cognition, 22, 1-13.

ToDD, J. J., \& MARoIs, R. (2004). Capacity limit of visual short-term memory in human posterior parietal cortex. Nature, 428, 751-754.

Treisman, A. M., \& Gelade, G. (1980). A feature-integration theory of attention. Cognitive Psychology, 12, 97-136.

Vogel, E. K., Woodman, G. F., \& Luck, S. J. (2001). Storage of features, conjunctions, and objects in visual working memory. Journal of Experimental Psychology: Human Perception \& Performance, 27, 92-114.

Wheeler, M. E., \& Treisman, A. M. (2002). Binding in short-term visual memory. Journal of Experimental Psychology: General, 131, 48-64.

Woodman, G. F., \& LUCK, S. J. (2004). Visual search is slowed when visuospatial working memory is occupied. Psychonomic Bulletin \& Review, 11, 269-274.

Woodman, G. F., \& Vogel, E. K. (2005). Fractionating working memory: Consolidation and maintenance are independent processes. Psychological Science, 16, 106-113.
Woodman, G. F., Vogel, E. K., \& Luck, S. J. (2001). Visual search remains efficient when visual working memory is full. Psychological Science, 12, 219-224.

\section{NOTES}

1. All of the VWM tasks in this article required memory for the conjunction of color and location. Similar tasks have been used by Fougnie and Marois (2006) and Cowan and Morey (2007). VWM tasks that require memory for feature bindings are the most likely to show dual-task costs between encoding/retrieval and storage, given the evidence that attention may be necessary for both the formation of feature bindings (Treisman \& Gelade, 1980) and their maintenance in WM (Fougnie \& Marois, 2009).

2. We found quantitatively similar correlation results using accuracy or capacity $(K)$ to measure the participants' single-task performance.

(Manuscript received January 20, 2009; revision accepted for publication June 27, 2009.) 